\title{
Los anticonceptivos con drospirenona, levonorgestrel u otros progestágenos se asociaron a un riesgo similar de eventos adversos serios
}

\author{
Drospirenone, levonorgestrel and other progestin containing oral contraceptives showed a similar risk \\ profile of serious adverse events
}

\section{Objetivo}

Investigar los riesgos a corto y largo plazo de la utilización de anticonceptivos orales (ACO) con un régimen de 24 días de drospirenona y etinilestradiol, comparados con los anticonceptivos orales ya conocidos.

\section{Diseño y lugares}

Estudio de cohorte prospectiva, controlada, no intervenida, abierta, realizado en 2.285 centros de prescripción de anticonceptivos orales en EE.UU. y seis países europeos (Austria, Croacia, Alemania, Italia, Polonia y Suecia).

\section{Pacientes}

Se enrolaron 85.109 mujeres que iniciaban, cambiaban o reiniciaban tratamiento con ACO. Al ingresar completaban un cuestionario acerca de su estado de salud (medicación, uso de $\mathrm{ACO}$ ) y factores de riesgo cardiovascular, que luego era revisado por el médico.

Se realizó el seguimiento por dos a seis años, lo que generó una observación de 206.296 mujeres por año. Hubo un 3,3\% de pérdidas de seguimiento.

\section{Intervención}

Se conformaron tres cohortes principales: regímenes de ACO que con-

Dinger J. y col. Contraception. 2014;89(4):253-63. tenían drospirenona de 24 días (DRSP-24d), de 21 días (DRSP-21d), y anticonceptivos que no contenían drospirenona (no-DRSP). A partir de éstos últimos se analizó una sub-cohorte de regímenes que contenían levonorgestrel (LNG).

\section{Medición de resultados principales}

Se calcularon los eventos adversos graves (los que resultan en la muerte, ponen en peligro la vida, requieren internación, generan discapacidad y/o requieren intervención quirúrgica) y se le dio especial interés al tromboembolismo venoso (TEV).

Cada reporte de efecto adverso era evaluado por un grupo de médicos en el "Berlin Center for Epidemiology and Health Research" que estaba ciego a la rama de tratamiento. Las conclusiones acerca de los efectos adversos se reportaron según el tratamiento recibido, y no por intención de tratar, ya que los resultados obtenidos fueron similares. Se realizó un ajuste por confundidores basado en modelo de expertos.

\section{Resultados principales}

En total se reportaron 5.964 eventos adversos serios y 162 eventos de TEV con incidencias similares en las cohortes principales (ver tabla 1).

Tabla 1: Incidencia y riesgo de eventos adversos serios y de tromboembolismo venoso según la cohorte estudiada de anticonceptivo oral.

\begin{tabular}{c|c|c} 
Sub-cohorte & \multicolumn{2}{c}{$\begin{array}{c}\text { Incidencia (IC 95\%) } \\
\text { eventos/10.000 mujeres-año }\end{array}$} \\
$\begin{array}{c}\mid c \\
\text { Eventos adversos serios }\end{array}$ & $\begin{array}{c}\text { Hazard radio } \\
\text { DRSP-24 vs comparador (IC 95\%) }\end{array}$ \\
\hline DRSP-24d & 248,4 & - \\
\hline no-DRSP & 262,3 & $0,9(0,8$ a 1,0$)$ \\
\hline LNG & 271 & - \\
\hline Tromboembolismo venoso** & \\
\hline DRSP-24d & $7,2(4,3$ a 11,2$)$ & $0,8(0,5 \text { a } 1,3)^{*}$ \\
\hline no-DRSP & $9,6(7,8$ a 11,6$)$ & $0,8(0,4 \text { a } 1,6)^{*}$
\end{tabular}

DRSP-24: regímenes de anticonceptivos orales con 24 días de drospirenona. No-DRSP: regímenes de anticonceptivos orales sin drospirenona. LNG: regímenes de anticonceptivos orales con levonorgestrel. * Ajustado por: edad, índice de masa corporal, duración del tratamiento con anticonceptivos orales y antecedentes familiares de TEV. ${ }^{* \star}$ En las no usuarias de anticonceptivos orales se registraron 3,9 casos/10.000 mujeres-año.

\section{Conclusiones}

Los anticonceptivos que contienen drospirenona tienen riesgos similares de eventos adversos serios, en particular TEV, en comparación con los que no contienen drospirenona o con- tienen levonorgestrel.

Fuentes de financiamiento/conflicto de intereses: Financiado por un productor de anticonceptivos orales (Bayer AG, Alemania).

\section{Comentario}

A pesar de que la incidencia del tromboembolismo venoso en las mujeres en edad reproductiva es bajo (3/10.000 mujeres-año) $)^{2}$, es sabido que la utilización de anticonceptivos orales aumenta el resgo y el efecto es considerable debido a la gran cantidad de mujeres que los utilizan.

Teniendo en cuenta que la hipótesis nula planteada en el diseño de este estudio fue un HR mayor o igual a dos, este trabajo demuestra que los ACO que contienen drospirenona tienen hasta el doble de riesgo o menos de TEV que los que no contienen drospirenona. Esto marca una ligera diferencia con respecto a estudios previos que adjudican un riesgo de hasta cuatro o seis veces mayor a los anticonceptivos con drospirenona en comparación con los de levonorgestrel. Sin embargo, el intervalo de confianza es considerablemente amplio como para per- mitirse asumir que los riesgos son similares.

Otro dato a destacar es la distribución de los factores de riesgo entre las cohortes. La cohorte de DRSP-24d presentaba menos pacientes fumadoras que la cohorte de LNG $(19,6 \%$ vs $25,2 \%)$ y menos pacientes con antecedentes de trombosis venosa profunda que la cohorte de no-DRSP $(0,06 \%$ vs. $0,13 \%)$.

\section{Conclusiones de la comentadora}

A la hora de elegir el tipo de anticonceptivos a prescribir es pertinente tener en cuenta el mayor riesgo de estos eventos con los que contienen drospirenona en comparación con los que contienen levonorgestrel, pero esta diferencia no parecería ser mayor al doble.

Paula Riganti [ Servicio de Medicina Familiar y Comunitaria del Hospital Italiano de Buenos Aires, paula.riganti@ hospitalitaliano.org.ar ]

Riganti P. Los anticonceptivos con drospirenona, levonorgestrel u otros progestágenos se asociaron a un riesgo similar de eventos adversos serios. Evid Actual Pract Ambul. 2015;18(3):85. Jul-Sep. Comentado de: Dinger J, y col. Cardiovascular and general safety of a 24-day regimen of drospirenone-containing combined oral contraceptives: final results from the International Active Surveillance Study of Women Taking Oral Contraceptives. Contraception. 2014;89(4):253-63. PMID: 24576793. 